\title{
AN EFFICIENT APPROACH TO IMPLEMENT FEDERATED CLOUDS
}

\author{
PAPPU SOWMYA, KUMAR R
}

Department of Computer Sciences, School of Computer Science and Engineering, VIT University, Chennai - 600 0127 , Tamil Nadu, India.

Email: asha.s@vit.ac.in

Received: 23 January 2017, Revised and Accepted: 03 March 2017

ABSTRACT

Cloud computing enables companies to several attractive benefits for business deals, in which software applications depend on the cloud storage centers, service providers, resource allocators, data servers, and many processing models; while selecting multiple service providers for computing resources, it improves performance, cost reduction than single cloud service provider. Federated cloud, also a generic term, is a coordination between the delegations of producer service cloud customer. While it satisfies efficiency, cost-effective, collaboration with service providers, it reduces overall management and infrastructure cost so that consumers can get services with less cost and high resources. In multi-cloud, the sharing of resources from service provider to consumer in an efficient way in federated cloud is a critical task. Achieving the cloud management while adding the multiple clouds. In this paper, the vendor lock-in problem of consumer and service provider getting resources with various processing levels, different methods and challenges in federated cloud architecture are provided in a specified study.

Keywords: Federated cloud, Multi-cloud, Resource provisioning, Public cloud.

(C) 2017 The Authors. Published by Innovare Academic Sciences Pvt Ltd. This is an open access article under the CC BY license (http://creativecommons. org/licenses/by/4. 0/) DOI: http://dx.doi.org/10.22159/ajpcr.2017.v10s1.19543

\section{INTRODUCTION}

Cloud federation is a new paradigm for achieving reliability, under- utilized, guaranteed availability, efficiency, and quality using federation and interconnected clouds. Interconnected clouds are nothing but using the private and public cloud. The cloud services are classified into three types. They are (1) Infrastructure as a Service (IaaS), (2) Platform as a Service (PaaS), and (3) Software as a Service (SaaS). However, federated cloud is a connection between public cloud and private cloud.

In federated cloud computing, the business, which has a group of service providers, consumers, and end-users, needs more access to providers and consumers who access the services available in the environment. The many cloud providers are available in the environment, i.e., European Grid Infrastructure [1], Amazon EC2 (Amazon Elastic Compute) [2], Rack-space (Cloud Computing and Hosting) [3] Microsoft Azure (developing and storing data) [4]. From this, data centers are the resources for service providers which can own their providing of the data, the accessing and utilizing the resources are customers, the services that are providing for end-users those can access the resources at anytime and anywhere.

The federated cloud that provides the accessing of resources from various services which have an agreement between service provider and customer to access the data is called as service level agreement. Federation cloud provides to build new business deals with clouds, where service providers can buy or sell the resources with efficient computing and relevant sources are available than other sources. Federated cloud acts a major role in the deployment models in the business deals.

The phenomenon that is used in federation which has the several terminologies provides the same thing which can be defined in three ways. They are (1) cloud federation, (2) inter-clouds, and (3) multiclouds. The term cloud federation is a collection of groups that have providers that combine mutual relation with each other and share the resources and provide the improvement services to the users [5]; they allow the virtual applications to be implemented in any federated clouds and give the location free to migrate in any part in between the sites those stored in data servers so that federation cloud is defined as the union of clouds to get the benefit from a particular form of business [6]. The term inter-cloud has the market-oriented size of clouds and shows the global exchange of cloud market-oriented are considered, the business-oriented federated cloud services have on time, scalable, efficient application services promoting in IaaS; the inter-cloud provides the services to resource mapping, cost optimization, quality of service, combining different sources, scalable resources are to be presented in flexible way of exchanging the producers and consumers to specific cloud coordinator with brokering agents. The final term multi-cloud provides the independent multiple clouds which can share the providers, end-users, and infrastructure clients those can manage resource scheduling and provisioning that has interoperability solutions on low-level functionality of clouds. Multi-cloud has identified the virtual mobility (VM) of sharing the resources [7]. CLOUD STACK SERVICE ARCHITECTURE

\section{Federation of clouds}

Federated cloud architecture is mediated by brokers with independent clouds of a vertical stack layer of services that those are from at the top is SaaS (software or application), in the middle PaaS (platform), at the bottom IaaS (infrastructure and operating system). In which each layer has fulfilled the request through the resources using delegation and by cloud providers is federation. Public or private cloud providing the business roles is in SaaS those request to the application layer where local resources are sufficient to provide available to service the requests in the same service, while they produce the services and goods that are in lack of efficient software interfaces and insufficient to provide considered sharing and market-oriented at the service layer (Fig. 1)

An application layer has a second thing that can increase the capacity through delegation, which works together providing the computing needs. In delegation, pay-as to-go models the PaaS layer and application layer in the cloud for provided more additional resources that can accessible in the different ways of depending on the current cloud. A request for more virtual machines can acquire from another cloud 


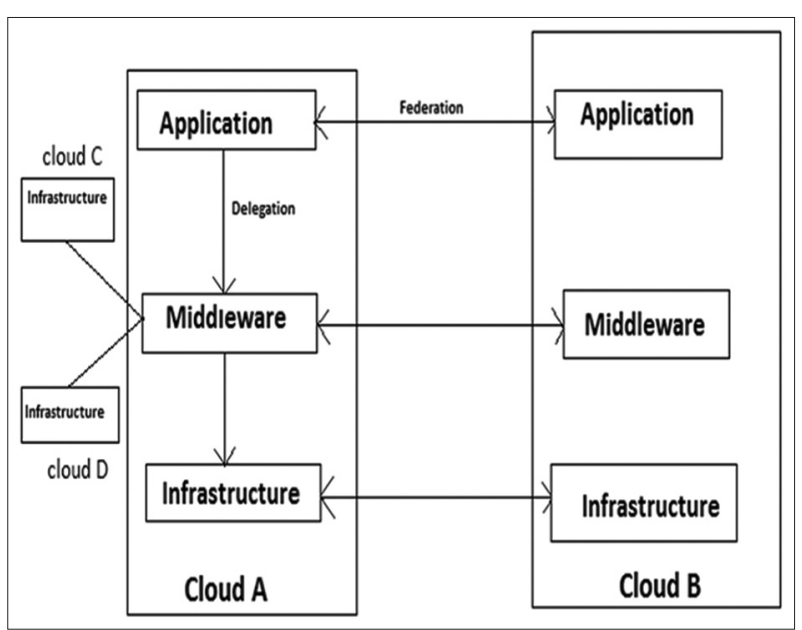

Fig. 1: Cloud application support in federation and delegation

in the federation though the brokering architecture available locally can attempt to acquire them from cloud federation. In general view of federation, which supports programs and environments between clouds and federations that contribute from infrastructure layer.

The federated cloud model assigns the emerging technologies for the work efficient. SaaS application gives a better result to the consumer when the application needs the detail process of the resource that can be meeting in delivery time in the fulfilled task of the service delivered to a customer. While understanding the federated cloud which has some critical work, because the architectures and patterns should have to be constructed in the parallel and grid computing for learning and switching into the different concepts to learn federated cloud scenario is slighter difficult work [8].

First, defining the different federated cloud methods allows the faulttolerance and elastic changes at different layer.

Second, service providers as well as consumers focus on the implement without having specific concrete methods in the full layer stack. Each of the different layers should provide the managing and corresponding data, processing the request from the user, which has to be performed in the account of cost and using specific computational requirements in the certain service.

\section{Federated architectures}

Coupling level of resources can be using architectures those can be promoting the resources based on public cloud and private cloud, those working schemes on the different basis where the consumer and provider interaction between the sharing of resources in the cloud with their workloads on which the federated clouds. The level of coupling with clouds can access security, migrating VMs, cloud instances, resource control in the cross-site networks.

\section{Cloud broker architecture}

Selecting and combining different cloud services and providers for cloud consumers are too complex, consumers are contracting cloud broker for services directly, and brokering agents act as a middleware for both consumers and providers to provide their specific needs of the product. The customer and provider contribute their workload in the multiple cloud with effective cost and deploying and managing the certain complexities of the required task. Customers can provide the application of a program and specific constraints with utilizing cloud services by ordering the prototype of the specific cloud provider (Fig. 2).

Consumer A and consumer B modules

The customers can access the resources from the applications, where the brokering agents provide the resources as different components.
The middleware provides the processing of task that should be accessing as an event handling, i.e., internal and external event and processing the task promotes to the service broker. The brokering agent has repository that stores the information about each and every task performed in the system.

The enterprise service broker module

The interaction with the consumers and service components that satisfy the needs of the customer needs. Each and every process to that will registry on the system, governance policy, should have the set of rules that can be performed for application which has accessing the resources.

The infrastructure as a service module

Which has to be cloud computing, there are different ways the infrastructure can be deployed. The infrastructure will depend on the application program and environment; however, the provider has chosen to build the cloud solution, which the number of servers required far, exceeds your desire budget to run those in-house.

\section{Cloud bursting architecture}

Using the computational resources of the private cloud or datacenter with their own internal meets the demand of the resource can be utilized on the basis of data. Each and every organization has a datacenter which is based on the VMware [9], to access the information and modify the information that can be store in the specific security. The consumers that have the many cloud providers, e.g., CloudSwitch [10], which has the isolation technology of cloud, which has the message access control and internet protocol of an internal network which appears on the public cloud.

\section{Multi-site cloud architecture}

Many number of service providers and consumers in the cloud which has a wide range of geographically distributed cloud that controls over the single cloud to the consumers, where they can migrate the live on cross-site networking and perform the VMware architectures in the cloud environment.

\section{Federated cloud technologies}

The technologies that support the federation and cloud services and implementing tools to construct the cloud environment those are:

\section{OpenNebula [11]}

OpenNebula provides an open source and simple feature with high flexible solution which can manage the virtual datacenters that can capable to access the turnkey enterprise-ready solution that needs to provide public, private, and hybrid cloud in the IaaS. OpenNebula can use the resources of its interoperability, leveraging existing information technology assets, protecting the deals, adding the application programming interface (API), facilitate to inter-cloud and closing vendor lock-in.

\section{Eucalyptus [12]}

Eucalyptus is an open source framework that performs the storage, network, and many other computational resources to access the cloud environment like OpenNebula; it allows for changes to the existing API for different resources. Eucalyptus implements in the Amazon Web Service (AWS) API facilitates interoperability with services and tools on compatible with the AWS API (amazon platform) that can be utilized on the federation for IaaS. Eucalyptus defines the pooling computational, storage, and network resources that can be measured scaled up or down as application workloads change in the utilization of the software. Eucalyptus systems declared a formal agreement like software license agreement with AWS in March 2012 to maintain compatibility.

\section{Aneka coordinator [13]}

Aneka coordinator is a resource management and deploying tool used in an Aneka enterprise cloud, to interacting and promoting resources 


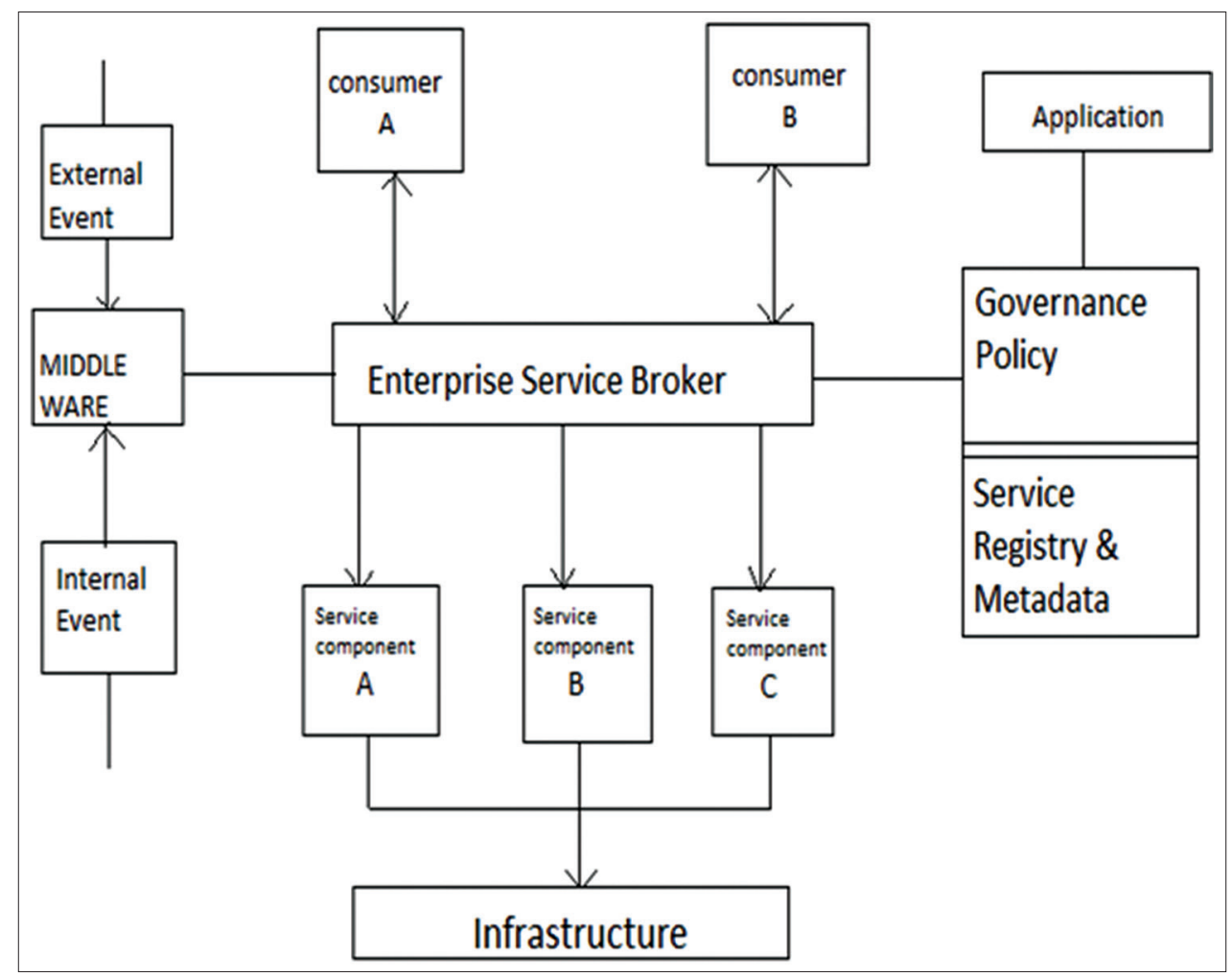

Fig. 2: An alternative model of federation cloud

with other Aneka sites. The Aneka coordinator is proposition of the Aneka services and Aneka peer components (network architectures) which provides the cloud ability and performance to interact with other cloud services. Aneka services provides multiple functional resources for providing peer-to-peer scheduling and peer-to-peer execution with accessing different jobs, while the Aneka peer facilitates resource sharing, cloud bursting, and load balancing among the distributed Aneka enterprise clouds those can be implement in a centralized and decentralized IaaS federation.

\section{CLOUD FEDERATION CHALLENGES}

Cloud consumers can be accessing the resources with provisioning of the computational needs. Combination of different clouds provides and the resources in the competitive task and schedules, while using the effective utilization of resource in required period of time. The challenges that are facing while using the resources from the cloud are as follows:

\section{Application architecture}

Deploying the resources in the considered service of an application which has the selected resource constrains that are less independent and promoting the more components on a single cloud.

\section{Deployment plan}

Cloud providers provides in the federated cloud which has different services, pricing models and service quality, to reduce the cost of the resource and increase the performance such as efficiency, responsiveness of a product. Locality of data servers provides the on time delivery. The model can be opted for gaining the final assessment for the delivering the output for the end-users, i.e., cloud customer aspects. The entire process of the work based on the delivery of the service.

\section{Specific constraints of the user}

User gives the different constraints that are to be provided for specific task that can be helpful to develop the application that can access with restricted decisions, while affinity constraints that has two or more
VMs are applied in same server or cluster, that applications has the local constraints that are used in the IaaS [14].

\section{Regulations, rules, and jurisdiction}

Cloud providers are from worldwide to share the resources and get the profitability from the customers with certain physical conditions. Hence, customer can implement the applications on different cloud providers, which has comply on regional laws to get the services on particular federal or state of a provenance.

\section{CLOUD FEDERATIONS INTEROPERABILITY ISSUES}

Interchangeability of different number of user applications is performed in different clouds and service providers that can be participating in a cloud federation represents and open issue, but it also has several open inter-related interoperability problems concerning the management and deployment of a large distributed and grid ecosystem. The European Commission provides the expert group that analyzes the challenges in the cloud research.

\section{Manageability}

Most of the cloud solutions handle elasticity, scalability, availability, efficient work; intelligent methodologies are provided to reach optimal resource utilization. Most data flowing or creating in the cloud require to be supported by meta-data information and technical standards are needed to guarantee the storing of resource and sharing of information among multiple cloud providers.

\section{Security and privacy}

Issues of data distribution and reliability of accessing should be in good addressed way to access, and security provides the high standards while resource sharing and providing among multiple tenants should be eliminated.

\section{Interoperability and federation}

The data structures should be replaced by high standards, and new approaches are provided to ensure combination toward interoperability 
avoiding vendor lock-in.

\section{Adaptability and virtualization}

Optimized resource-providing solutions are assigned to be considering for cross-cloud platform for executions, migrations in the different areas of taking into an application providing workloads.

\section{Programming models and economy}

High control on data distribution and resources should be companied, and innovative methods are assigned for application, monitor, development, and deployment, which are promoted for resource utilization, more efficient resource utilization with reduced power consumption.

\section{IMPLEMENTATION WORK}

Cloud customers use OpenNebula to virtualize the data center, integrate proposed information technology assets for storage, network, and computing with consolidate servers. In this model, deployment can do directly with OpenNebula which hypervisor is used as VMware ESXi to complete control over physical and virtual sources, which are enhancing advanced technologies for business continuity and high availability for optimizing the resource. Cloud management provides the more features while adding to federate datacenters, implementing the different cloud software to implanting the cloud bursting and offers self-service pattern for users.

Consider OpenNebula is installed on one physical machine with using of the ESXi hypervisors, the deployment model, the cloud brokers are vOneCloud is distribution optimization for an OpenNebula to work on VMware product. The multiple clouds that need to register like vOneCloud as to be the providing of the specific task that can be analyzing for adding the clouds on clouds. The cloud environment that has to be provided specific username and password for each and every client machine to access the clouds, here in which Vsphere Webclient, and the different cloud Admin, OneAdmin like users are there in the Vcenter directory. The system OpenNebula is installed in Centos 7 operating system (Fig. 3) [15].

The Vsphere Webclient is installed on the Windows server, and in that, the cloud provider that has the hybrid deployment of OpenNebula is installed.

When we power on the machine, it has to be open the OpenNebula cloud environment, where multiple clouds are installed and in which the images of different operating systems are there to specify the communication between different data centers, hosts, and network configuration are to be specified. If we want to create more instances in the OpenNebula system, we use the different options on the Vsphere Webclient, i.e., create VMs with storage, network configuration, and computing aspects of different options, those virtual machines can be stored in the Vsphere cloud directory; later, we created the virtual machines that can be login with the local host with minimal version of Centos 7 operating system. When we get import to our system, i.e., OpenNebula Sunstone dashboard will appear in that virtual machines, hosts, clusters, datacenters, zones which are provided in infrastructure; for each and every virtual machine, it shows the number of users and memory allocated, space occupied for each machine, load balancing in the environment.

In the dashboard, the specific type of machines is to be running of the different hosts and systems that can be provided for configuring the number of machines in it. The command line interface for the configuring the tools in the machines that can be assigned for specific number of machines that can be rectified by the certain error-based commands to configure the process.

\section{RELATED WORK AND FUTURE ENHANCEMENT}

Cloud computing and federated cloud are a new emerging technology,

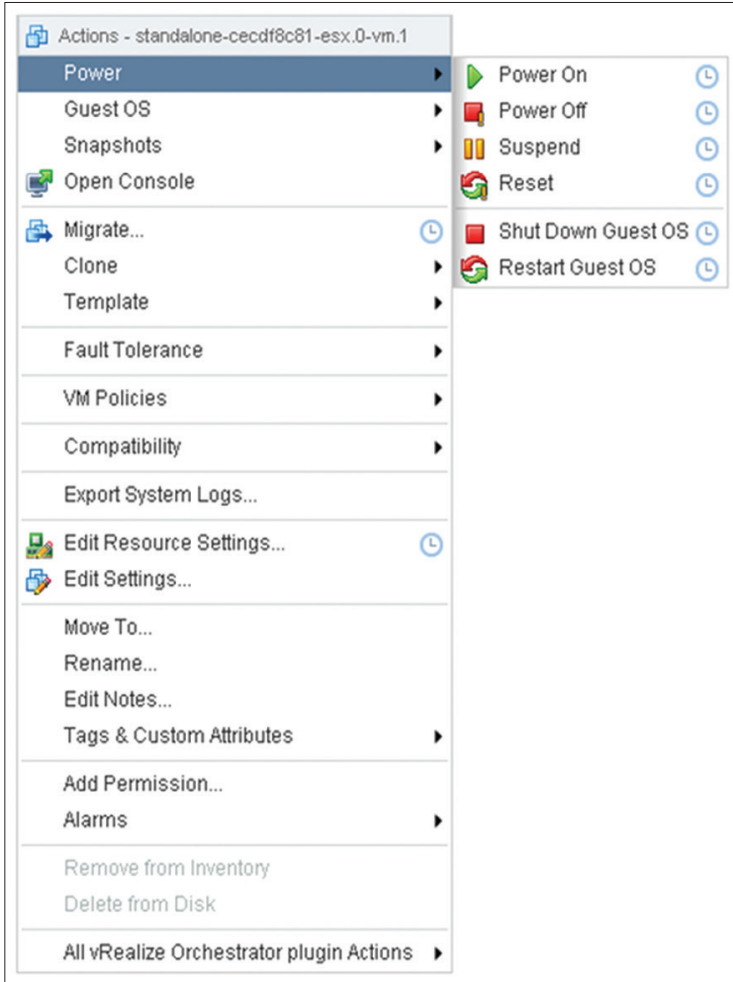

Fig. 3: Vsphere webclient dashboard

which are used in the business deals which can management and deployment of multiple internal and external cloud computing services. When a large collection of cloud providers and servers connect their infrastructure to provide resources that can be shared among other services in a reliable agreement. A new methodology can enhance the scalability and reliability for constructing large scalable and efficient resources that are provided and discovered in cloud federation. The most popular conflicting goals of the user and cloud providers have a demand on system policies, objectives, and best trade-off. The general method to the formation and issues of cloud federations has a distributed, heterogeneous environment on both the commercial and academic area.

COMPARING RESULTS

Evaluating the number of resources in the system that can be cloud environment, which on running on OpenNebula instances occupies the more space to run single instance in one virtual machine. In the same case, OpenNebula is easy to implement and where the OpenStack instances take the less space. The configuring the specific machine is difficult in OpenStack. The memory and central processing unit allocation need more space to run the process, where the memory size is $1 \mathrm{~TB}$, even though for running with number of hosts, virtual machines, datacenters, zones, etc., those require the space. Each single resource occupies the approximately 3 GB of memory space.

\section{CONCLUSION}

The main goal of the participating providers, servers, end-users is mainly for resource utilization and reducing costs. While users are mostly focus on optimizing the resource performance of a single application or workflow, we have to solve the problems regarding negotiation process and delegation process of the particular service using the different cloud services. These multi-cloud infrastructures are used to avoid provider lock-in issues for end-users that frequently utilize different clouds and servers. We have surveyed and characterized recent solutions and research on that attempt to hide the diversity of multiple clouds and 
single cloud those can form a unified federation on top of them, but they still need to scope with several open issues. In federated cloud is made where the basic architectures and the challenges that are associated with promoting of resources are discussed. Finally, existing solutions and research on resource sharing in federated cloud by evaluating their management, monitoring, evaluating, allocating resources, deployment are analyzed to give better results.

\section{REFERENCES}

1. European Grid Infrastructure. Available from: http://www.egi.com/ resources/. [Last accessed on 2015 Oct 10].

2. Amazon Elastic Compute Cloud; 2015. Available from: http://www. aws.amazon.com/EC2/resources. [Last accessed on 2015 Aug 25].

3. Open Cloud Computing and Hosting Servers, Rackspace. Available from: http://www.rackspace.com/. [Last accessed on 2015 Sep 5].

4. Windows Azure: Microsoft's Cloud Platform, Hosting, Developing, Services; 2015. Available from: http://www.windowsazure.com/. [Last accessed on 2015 Jul 25].

5. Kurze T, Bermbachy D, Klemsy M. Cloud federation. In: Proceedings of the $2^{\text {nd }}$ International Conference on Cloud Computing GRIDs, and Virtualization. Vol. 3. 2011. p. 32-8.

6. Kayed A, Shareef O. Survey on federated clouds. In: Proceedings with International Journal of Advanced Research Paper. Vol. 5. No. 2. February; 2015.

7. Diamond S, Morrow M, Bernstein D. Blueprint for the Inter-cloud. In: Proceedings of the Fourth International Conference on Internet and Web Applications and Services. Vol. 9. 2009. p. 328-36.

8. Abawajy J, Buyya R, Beloglazov A. Energy efficient management of data center resources for cloud computing. In: Proceedings with
International Conference on Parallel and Distributed Processing Techniques and Application. Las Vegas, USA; 2010.

9. VMware Virtualization Software for Desktops, Servers. Available from: http://www.vmware.com/. [Last accessed on 2015 Jul 20].

10. Enterprise Cloud Computing - Cloud Switch. Available from: http:// www.cloudswitch.com/resources/. [Last accessed on 2015 Sep 10].

11. Resource leasing and suspending virtual machines. A characterised on federated clouds. Proceedings on $11^{\text {th }}$ IEEE International Conference on High Performance Computing and Communications; 2009. p. 59-68. Available from: http://www.opennebula/onevcloud.com/resources.

12. Nurmi D, Wolski R, Obertelli G, Soman S, Zagorodnov D. The eucalyptus open-source cloud-computing system. In: Proceedings with International Symposium on Cluster Computing and Grid. Shanghai, China; 2009. Available from: http://eucalyptus.com/resources.

13. Kim H, Chaudhari S, Parashar M. Aneka cloud infrastructure and online risk analytics on the cloud. In: Proceedings of the $9^{\text {th }}$ IEEE/ACM International Symposium on Cluster Computing and the Grid. 2009. p. 484-9. Available from: http://www.aneka.org/networks/.

14. Montero RS, Llorente IM. IaaS cloud architecture: Virtualized datacenters to federated cloud infrastructures. Computer 2012;45(12):65-72.

15. Operating System with Linux Environment. Available from: http:// www.centos.org/about/isoimages.

16. Available from: http://www.computenext.com/blog/what-is-therelationship-between-hybrid-clouds-and-federated-clouds.

17. Mohmood Z. Cloud Computing-Challenges, Limitations and R\&D Solutions. Available from: http://www.springer.com/chapter/10/pages 277 to 296.

18. Rochwerger B. An architecture for federated cloud computing. ;25(6):599-616. 